\title{
Asymptotic Behavior of Multigroup SEIR Model with Nonlinear Incidence Rates under Stochastic Perturbations
}

\author{
Feng Wang $\mathbb{D},{ }^{1,2}$ Shan Wang $\mathbb{D},^{2}$ and Youhua Peng $\mathbb{D}^{2}$ \\ ${ }^{1}$ School of Mathematics and Statistics, Central South University, 410083 Changsha, China \\ ${ }^{2}$ Department of Mathematics, Pingxiang University, 337000 Pingxiang, China \\ Correspondence should be addressed to Youhua Peng; pengyouh@126.com
}

Received 26 November 2019; Accepted 20 April 2020; Published 5 May 2020

Academic Editor: Carmen Coll

Copyright (c) 2020 Feng Wang et al. This is an open access article distributed under the Creative Commons Attribution License, which permits unrestricted use, distribution, and reproduction in any medium, provided the original work is properly cited.

In this paper, the asymptotic behavior of a multigroup SEIR model with stochastic perturbations and nonlinear incidence rate functions is studied. First, the existence and uniqueness of the solution to the model we discuss are given. Then, the global asymptotical stability in probability of the model with $R_{0}<1$ is established by constructing Lyapunov functions. Next, we prove that the disease can die out exponentially under certain stochastic perturbation while it is persistent in the deterministic case when $R_{0}>1$. Finally, several examples and numerical simulations are provided to illustrate the dynamic behavior of the model and verify our analytical results.

\section{Introduction}

The history of human beings is full of struggle against diseases which cause great disaster to humans. At present, many countries and people around the world are suffering from the COVID-19, which has seriously affected people's lives and brought huge losses to the economy. Epidemiology is the subject to study the spread of diseases and formulate the strategies and measures for controlling and eliminating diseases. Mathematical modeling has been widely used in epidemiology to depict the mechanism of disease transmission and study the behavior of disease. One of the classic epidemic models is the SIR model which divides the host population into three parts, the susceptible, the infective, and the removed, and records their sizes by $S(t), I(t)$, and $R(t)$ at time $t$, respectively. However, many diseases do not break out immediately, and there will be a latent period of time, so SEIR models with latent period have been widely studied. In SEIR models, the size of the exposed individuals is labeled by $E(t)$ at time $t$.

Many models have considered the case of only one group; however, groups in different communities, regions, or with different cultural backgrounds have various lifestyles, dietary habits, and so on, which will make the disease have different ways of transmission. Therefore, considering different contact patterns, transmission, or geographic distributions, it is more reasonable to divide the host population into several subgroups and study the disease interactions among different subgroups. This is known as the multigroup model. One of the earliest works on the multigroup disease model was done by Lajmanovich and Yorke [1], who discussed a class of SIS multigroup models for the transmission of gonorrhea and used Lyapunov functions to prove the stability of the unique endemic equilibrium. Since then, there has been a great quantity of literature on the multigroup model, such as [2-8].

In the classic SEIR models, the incidence function takes the bilinear form. A premise for this form is that the host population is homogeneously mixed, and everyone has the same possibility to be infected when the infectives are introduced to the group. In real life, however, the population may not be homogeneously mixed, and the immunity of each person may be different such that the chances of being infected are disparate, so extending bilinear incidence to nonlinear functions can conform to the actual situation better. Many scholars have studied the epidemic models with nonlinear incidence rate, such as $[4,8-11]$ and the reference therein. Also, many scholars investigated the epidemic 
models with time delays, such as $[12,13]$. In [4], the authors discussed the global stability of the multigroup epidemic model with nonlinear incidence rates of the form $f_{k j}\left(S_{k}, I_{j}\right)$, which satisfies the following assumptions:

(i) (H1) for $0<S_{k} \leq S_{k}^{0}$, it has $0<\lim _{I_{j} \longrightarrow 0^{+}}$ $\left(f_{k j}\left(S_{k}, I_{j}\right) / I_{j}\right)=C_{k j}\left(S_{k}\right)$, where $S_{k}^{0}$ is the positive solution of certain function.

(ii) (H2) $f_{k j}\left(S_{k}, I_{j}\right) \leq C_{k j}\left(S_{k}\right) I_{j}$ for any $I_{j}>0$.

(iii) $(\mathrm{H} 3) C_{k j}\left(S_{k}\right) \leq C_{k j}\left(S_{k}^{0}\right)$, for $0<S_{k} \leq S_{k}^{0}$.

This research intends to study this general form of incidence function and assumes further that $\left(C_{k j}\left(S_{k}\right) / S_{k}\right) \leq K$, where for $K$ is a positive constant. The above incidence rate functions $f_{k j}\left(S_{k}, I_{j}\right)$ include some special cases which can be seen in some literature, for example,

$$
\begin{aligned}
& f_{k j}\left(S_{k}, I_{j}\right)=S_{k} I_{j}, \\
& f_{k j}\left(S_{k}, I_{j}\right)=S_{k}^{q} I_{j}, \quad q \geq 1, \\
& f_{k j}\left(S_{k}, I_{j}\right)=\frac{S_{k} I_{j}}{1+\alpha I_{j}^{2}}, \\
& f_{k j}\left(S_{k}, I_{j}\right)=\frac{S_{k} I_{j}}{\varphi\left(I_{j}\right)} .
\end{aligned}
$$

The multigroup SEIR model with above incidence functions can be obtained:

$$
\left\{\begin{array}{l}
\frac{d S_{k}}{d t}=\Lambda_{k}-\sum_{j=1}^{n} \beta_{k j} f_{k j}\left(S_{k}, I_{j}\right)-d_{k}^{S} S_{k}, \\
\frac{d E_{k}}{d t}=\sum_{j=1}^{n} \beta_{k j} f_{k j}\left(S_{k}, I_{j}\right)-\left(\epsilon_{k}+d_{k}^{E}\right) E_{k}, \\
\frac{d I_{k}}{d t}=\epsilon_{k} E_{k}-\left(\alpha_{k}+d_{k}^{I}+\gamma_{k}\right) I_{k} \\
\frac{d R_{k}}{d t}=\gamma_{k} I_{k}-d_{k}^{R} R_{k} .
\end{array}\right.
$$

What the parameters mean can be summarized in the following list:

$\Lambda_{k}$ : the influx of individuals in the $k$ th group.

$\beta_{k j}$ : the transmission rate between $S_{k}$ and $I_{j}$.

$d_{k}^{S}, d_{k}^{E}, d_{k}^{I}$, and $d_{k}^{R}$ : the natural death rate of $S, E, I$, and $R$ in the kth group, respectively.

$\epsilon_{k}$ : the rate of becoming infectious in the $k$ th group. $\alpha_{k}$ : the death rate caused by disease in the $k$ th group.

$\gamma_{k}$ : the cure rate in the $k$ th group.

The parameters above are all nonnegative. In particular, when $\beta_{k j}=0$, it means that there is no disease transmission between $S_{k}$ and $I_{j}$. The matrix $B=\left(\beta_{k j}\right)_{n \times n}$ reflects the transmission mechanism of disease among different subgroups built in the model. In this paper, we assume that the matrix $B$ is irreducible.

Since that $R_{k}, k=1,2, \ldots, n$, do not appear in the first three equations of model (2) but only in the fourth equation, their properties and behaviors can be solved easily if $I_{k}, k=1,2, \ldots, n$, are known; they can be omitted when analyzed. Therefore, the model can be simplified into the following form:

$$
\left\{\begin{array}{l}
\frac{d S_{k}}{d t}=\Lambda_{k}-\sum_{j=1}^{n} \beta_{k j} f_{k j}\left(S_{k}, I_{j}\right)-d_{k}^{S} S_{k}, \\
\frac{d E_{k}}{d t}=\sum_{j=1}^{n} \beta_{k j} f_{k j}\left(S_{k}, I_{j}\right)-\left(\epsilon_{k}+d_{k}^{E}\right) E_{k}, \\
\frac{d I_{k}}{d t}=\epsilon_{k} E_{k}-\left(\alpha_{k}+d_{k}^{I}+\gamma_{k}\right) I_{k} .
\end{array}\right.
$$

In the epidemic models, the basic reproduction number $R_{0}$, which represents the number of second generations produced by a single infected individual, plays an important role in the spread of disease for the long time. According to $[4,14], R_{0}=\rho\left(M_{0}\right)$, where $M_{0}=\left(\beta_{k j} \epsilon_{k} C_{k j}\left(S_{k}^{0}\right) /\left(\alpha_{k}+d_{k}^{I}\right.\right.$ $\left.\left.+\gamma_{k}\right)\left(\epsilon_{k}+d_{k}^{E}\right)\right)_{n \times n}, S_{k}^{0}=\Lambda_{k} / d_{k}^{S}$, and $\rho$ is the spectral radius of the matrix $M_{0}$. If $R_{0}<1$, there is only disease-free equilibrium $P_{0}$, where $P_{0}=\left(\left(\Lambda_{1} / d_{1}^{S}\right), 0,0, \ldots,\left(\Lambda_{n} / d_{n}^{S}\right), 0,0\right)$. When $R_{0}>1$, then $P_{0}$ is unstable, and the model has an endemic equilibrium $P^{*}$ which means the disease will be persistent. In this situation, our concern is whether there is a way to exterminate the disease.

The reality is filled with randomness, and the epidemic models are often influenced by random environments. For example, there are a lot of natural disasters in reality, such as storm and earthquake. If these randomnesses happen, the parameters and the transmission mechanism in the model are likely to be affected. Thus, the deterministic model has some limitations to fully describe transmission of disease. Many scholars have studied the epidemic model with stochastic perturbations depicted by Brownian motion, and a lot of literature studies have been published; we refer the readers to $[5,7,10,12,13,15-17]$. In [18-20], the authors studied the SIR or SIRS model with Markovian switching, and they gave some conditions on extinction or ergodicity of the model.

Influenced by the work of predecessors, we use the similar method of Dalal et al. and Witbooi [21, 22] to construct stochastic perturbations, that is, we replace the parameters $d_{k}^{E}$ and $d_{k}^{I}$ by $d_{k}^{E}-\sigma_{1 k} d B_{k}$ and $d_{k}^{I}-\sigma_{2 k} d W_{k}$, where the stochastic perturbations $B_{k}$ and $W_{k}$ are independent standard Brownian motions. The reason that not all parameters but only some of them are disturbed by stochastic perturbations may be the uncertainty of stochastic factors and the change of behavior of the infected.

For all we know, the papers that discuss asymptotic behaviors of stochastic multigroup SEIR models with nonlinear incidence rate functions are relatively few. In this paper, we will study the following stochastic multigroup SEIR model: 


$$
\left\{\begin{array}{l}
\dot{S}_{k}=\left(\Lambda_{k}-\sum_{j=1}^{n} \beta_{k j} f_{k j}\left(S_{k}, I_{j}\right)-d_{k}^{S} S_{k}\right) \mathrm{d} t, \\
\dot{E}_{k}=\left(\sum_{j=1}^{n} \beta_{k j} f_{k j}\left(S_{k}, I_{j}\right)-\left(\epsilon_{k}+d_{k}^{E}\right) E_{k}\right) \mathrm{d} t+\sigma_{1 k} E_{k} \mathrm{~d} B_{k}(t), \\
\dot{I}_{k}=\left(\epsilon_{k} E_{k}-\left(\alpha_{k}+d_{k}^{I}+\gamma_{k}\right) I_{k}\right) \mathrm{d} t+\sigma_{2 k} I_{k} \mathrm{~d} W_{k}(t),
\end{array}\right.
$$

where $\sigma_{i k}, i=1,2$, are the intensities of stochastic perturbation.

Because the incidence rate functions $f_{k j}\left(S_{k}, I_{j}\right)$ are general and can be of different types in one model, which increase the difficulty of research, we will overcome it by some inequality techniques. This paper is organized as follows. Section 2 presents some background knowledge and lemmas which will be used afterwards. In Section 3, we prove that there is a unique positive solution to the model for any initial value. Section 4 proves that the disease-free equilibrium is globally asymptotically stable in probability when $R_{0}<1$ by constructing Lyapunov functions. In Section 5 , the disease will die out exponentially under certain stochastic perturbations when $R_{0}>1$, and in Section 6, we provide some numerical simulations of the model to verify our analytical results.

\section{Preliminaries}

Throughout the paper, unless otherwise specified, $\left(\Omega,\left\{\mathscr{F}_{t}\right\}_{t \geq 0}, P\right)$ denotes a complete probability space with a filtration $\left\{\mathscr{F}_{t}\right\}_{t \geq 0}$ satisfying the usual conditions (i.e., it is right continuous, and $\mathscr{F}_{0}$ contains all $P$-null sets). Denote

$$
\mathbb{R}_{+}^{n}=\left\{x \in R^{n}: x_{i}>0 \text { for all } 1 \leq i \leq n\right\} .
$$

In general, let $X$ be a regular homogeneous Markov process in $R^{n}$; consider the stochastic differential equation

$$
\mathrm{d} X(t)=b(X(t)) \mathrm{d} t+\sum_{k=1}^{d} \sigma_{k}(X(t)) \mathrm{d} B_{k}(t),
$$

with initial value $X\left(t_{0}\right)=x_{0} \in R^{n}$ and $B_{k}(t), 1 \leq k \leq d$, are standard Brownian motions. Define the differential operator $\mathscr{L}$ associated with the above equation by

$$
\mathscr{L}=\sum_{k=1}^{n} b_{k}(x) \frac{\partial}{\partial x_{k}}+\frac{1}{2} \sum_{i=1}^{n} \sum_{j=1}^{n}\left[\sigma^{T}(x) \sigma(x)\right]_{i j} \frac{\partial^{2}}{\partial x_{i} \partial x_{j}} .
$$

If $\mathscr{L}$ acts on a function $V \in C^{2,1}\left(E^{l} \times \mathbb{R}_{+} ; \mathbb{R}_{+}\right)$, then by Itô formula,

$$
\mathrm{d} V(X, t)=\mathscr{L} V(X, t) \mathrm{d} t+\sum_{r=1}^{d} V_{x}(X, t) \sigma_{r}(X(t)) \mathrm{d} B_{r}(t) .
$$

where

$$
\begin{aligned}
\mathscr{L} V(X, t)= & V_{t}(X, t)+\sum_{k=1}^{n} b_{k}(x) \frac{\partial V}{\partial x_{k}} \\
& +\frac{1}{2} \sum_{i=1}^{n} \sum_{j=1}^{n}\left[\sigma^{T}(x) \sigma(x)\right]_{i j} \frac{\partial^{2} V}{\partial x_{i} \partial x_{j}} .
\end{aligned}
$$

Next, we introduce some definitions about stability and lemmas which will be used latter. Assume that $b(0)=0$ and $\sigma_{k}(0)=0, k=1,2, \ldots, d$; then, $X(t) \equiv 0$ is the trivial solution to (6).

Definition 1. The trivial solution is called to be

(i) Stable in probability if for any $\epsilon>0$ and the solution $X\left(t, x_{0}\right)$ with initial value $X(0)=x_{0}$, then

$$
\lim _{x_{0} \longrightarrow 0} \mathbb{P}\left(\sup _{t \geq 0}\left|X\left(t, x_{0}\right)\right| \geq \epsilon\right)=0 .
$$

(ii) Globally asymptotically stable in probability if it is stable in probability, and for any $x_{0} \in \mathbb{R}^{n}$,

$$
\mathbb{P}\left(\lim _{t \longrightarrow \infty} X\left(t, x_{0}\right)=0\right)=1 .
$$

Lemma 1 (cf. [23]). If there is a positive definite function $V(t, x) \in \mathscr{C}^{2}\left(\mathbb{R}^{n}\right)$ with an infinitesimal upper limit such that the function $\mathscr{L V}$ is negative definite, then the trivial solution is globally asymptotically stable in probability.

Lemma 2 (Perron-Frobenius). If $A=\left(a_{i j}\right)_{n \times n}$ is irreducible and nonnegative, then the spectral radius $\rho(A)$ of $A$ is a single eigenvalue, and there is a positive eigenvector $\omega=\left(\omega_{1}, \omega_{2}, \ldots, \omega_{n}\right)$ corresponding to $\rho(A)$ of $A$. Moreover, $\rho(A)$ satisfies the inequality

$$
\min _{i} \sum_{j} a_{i j} \leq \rho(A) \leq \max _{i} \sum_{j} a_{i j} .
$$

Remark 1. From our previous description in Introduction, we know that $R_{0}=\rho\left(M_{0}\right)<1$ will lead to the extinction of disease in deterministic model (3). Combining the expression of $R_{0}$ with the estimation of $\rho\left(M_{0}\right)$ in (12), we can infer that if transmission rate $\beta_{k j}$ decreases, $\rho\left(M_{0}\right)$ will become smaller, which provides the possibility of eliminating disease. A very important way to reduce $\beta_{k j}$ is to isolate people at home and restrict them from going out. This measure is being taken in many countries to combat COVID-19.

Lemma 3 (cf. [24]). Let $M=\left\{M_{t}\right\}_{t \geq 0}$ be a real-valued continuous local martingale vanishing at $t=0$. Then, 


$$
\begin{array}{cc}
\lim _{t \rightarrow \infty}\langle M, M\rangle_{t}=\infty & \text { a.s. } \Longrightarrow \lim _{t \rightarrow \infty} \frac{M_{t}}{\langle M, M\rangle_{t}}=0 \text { a.s. } \\
\limsup _{\mathrm{t} \rightarrow \infty} \frac{\langle M, M\rangle_{t}}{t}<\infty & \text { a.s. } \Longrightarrow \lim _{t \rightarrow \infty} \frac{\mathrm{M}_{\mathrm{t}}}{\mathrm{t}}=0 \text { a.s.. }
\end{array}
$$

\section{The Existence and Uniqueness of the Solution to Model (4)}

The first question we concern is whether the system has a solution or not. In this section, we prove that the system has a global and positive solution for any initial value.

Theorem 1. Given any initial value $\left(S_{1}(0), E_{1}(0), I_{1}(0)\right.$ $\left.\cdots S_{n}(0), E_{n}(0), I_{n}(0)\right) \in \mathbb{R}_{+}^{3 n}$, then model (4) has a unique solution on $t \geq 0$, and the solution will remain in $\mathbb{R}_{+}^{3 n}$ with probability one, that is, $\left(S_{1}(t), E_{1}(t), I_{1}(t) \cdots S_{n}(t), E_{n}(t)\right.$, $\left.I_{n}(t)\right) \in \mathbb{R}_{+}^{3 n}$ for $t \geq 0$ almost surely.

Proof. Since the coefficients of the model are locally Lipschitz continuous, there is a unique local solution $\left(S_{1}(t), E_{1}(t), I_{1}(t) \cdots S_{n}(t), E_{n}(t), I_{n}(t)\right) \quad$ on $\quad t \in\left[0, \tau_{e}\right]$, where $\tau_{e}$ is the explosion time (cf. [24]). In order to illustrate the solution is global, we only need to prove $\tau_{e}=\infty$. Assume $c_{0}$ is sufficiently large so that $S_{1}(0), E_{1}(0), I_{1}(0) \ldots$ $S_{n}(0), E_{n}(0), I_{n}(0)$ lie within the interval $\left[\left(1 / c_{0}\right), c_{0}\right]$. For $c \geq c_{0}$, define the stopping time

$$
\begin{gathered}
\tau_{c}=\inf \left\{t \in\left[0, \tau_{e}\right], \min _{1 \leq k \leq n}\left\{S_{k}(t), E_{k}(t), I_{k}(t)\right\} \leq \frac{1}{c},\right. \\
\left.\quad \text { or } \max _{1 \leq k \leq n}\left\{S_{k}(t), E_{k}(t), I_{k}(t)\right\} \geq c\right\} .
\end{gathered}
$$

We set $\inf \varnothing=\infty$ (where $\varnothing$ denotes the empty set). Clearly, $\tau_{c}$ is increasing as $c \longrightarrow \infty$. Let $\tau_{\infty}=\lim _{c \longrightarrow \infty} \tau_{c}$, and $\tau_{\infty} \leq \tau_{e}$ a.s. If we can prove $\tau_{\infty}=\infty$ a.s., then equality $\tau_{e}=\infty$ holds true, and the conclusion can be obtained. If the assertion is false, then there exist two constants $T>0$ and $\epsilon \in(0,1)$ such that

$$
\mathbb{P}\left(\tau_{\infty} \leq T\right)>\epsilon .
$$

Hence, there exists a positive integer $c_{1} \geq c_{0}$ such that

$$
\mathbb{P}\left(\tau_{c} \leq T\right)>\epsilon, \text { for all } c \geq c_{1} .
$$

Then, we define a function $V: R_{+}^{3 n} \longrightarrow R$ by

$$
\begin{aligned}
V\left(S_{k}, E_{k}, I_{k}\right):= & \sum_{k=1}^{n}\left[\left(S_{k}-a_{k}-a_{k} \ln \frac{S_{k}}{a_{k}}\right)\right. \\
& +\left(E_{k}-1-\ln E_{k}\right) \\
& \left.+\left(I_{k}-1-\ln I_{k}\right)\right],
\end{aligned}
$$

where $a_{k}, k=1,2, \cdots n$, are constants which will be determined later. Using Itồ's formula, we can get

$$
\begin{aligned}
\mathrm{d} V= & \sum_{k=1}^{n}\left(1-\frac{a_{k}}{S_{k}}\right)\left[\left(\Lambda_{k}-\sum_{j=1}^{n} \beta_{k j} f_{k j}\left(S_{k}, I_{j}\right)-d_{k}^{S} S_{k}\right) \mathrm{d} t\right] \\
& +\frac{1}{2} \sum_{k=1}^{n}\left[\sigma_{1 k}^{2}+\sigma_{2 k}^{2}\right] \\
& +\sum_{k=1}^{n}\left(1-\frac{1}{E_{k}}\right)\left[\left(\sum_{j=1}^{n} \beta_{k j} f_{k j}\left(S_{k}, I_{j}\right)-\left(\epsilon_{k}+d_{k}^{E}\right) E_{k}\right) \mathrm{d} t\right. \\
& \left.+\sigma_{1 k} E_{k} \mathrm{~d} B_{k}(t)\right] \\
& +\sum_{k=1}^{n}\left(1-\frac{1}{I_{k}}\right)\left[\left(\epsilon_{k} E_{k}-\left(\alpha_{k}+d_{k}^{I}+\gamma_{k}\right) I_{k}\right) \mathrm{d} t\right. \\
& \left.+\sigma_{2 k} I_{k} d W_{k}(t)\right] \mathrm{d} t \\
= & \mathscr{L} V \mathrm{~d} t+\sum_{k=1}^{n}\left[\sigma_{1 k}\left(E_{k}-1\right) \mathrm{d} B_{k}(t)+\sigma_{2 k}\left(I_{k}-1\right) \mathrm{d} W_{k}(t)\right],
\end{aligned}
$$

where

$$
\begin{aligned}
\mathscr{L} V= & \sum_{k=1}^{n}\left[\Lambda_{k}-d_{k}^{S} S_{k}-\frac{a_{k}}{S_{k}} \Lambda_{k}+\frac{a_{k}}{S_{k}} \sum_{j=1}^{n} \beta_{k j} f_{k j}\left(S_{k}, I_{j}\right)\right. \\
& +a_{k} d_{k}^{S}-d_{k}^{E} E_{k} \\
& \left.-\frac{1}{E_{k}} \sum_{j=1}^{n} \beta_{k j} f_{k j}\left(S_{k}, I_{j}\right)-\left(\alpha_{k}+d_{k}^{I}+\gamma_{k}\right) I_{k}-\frac{\epsilon_{k} E_{k}}{I_{k}}\right] \\
& +\epsilon_{k}+d_{k}^{E}+\alpha_{k}+d_{k}^{I}+\gamma_{k}+\frac{1}{2} \sum_{k=1}^{n}\left[\sigma_{1 k}^{2}+\sigma_{2 k}^{2}\right] \\
\leq & \sum_{k=1}^{n}\left[\Lambda_{k}+a_{k} \sum_{j=1}^{n} \beta_{k j} K I_{j}+a_{k} d_{k}^{S}-\left(\alpha_{k}+d_{k}^{I}+\gamma_{k}\right) I_{k}\right] \\
& +\epsilon_{k}+d_{k}^{E}+\alpha_{k}+d_{k}^{I}+\gamma_{k}+\frac{1}{2} \sum_{k=1}^{n}\left[\sigma_{1 k}^{2}+\sigma_{2 k}^{2}\right] .
\end{aligned}
$$

Notice that

$$
\begin{aligned}
\sum_{k=1}^{n} & \sum_{j=1}^{n} a_{k} \beta_{k j} K I_{j}-\sum_{k=1}^{n}\left(\alpha_{k}+d_{k}^{I}+\gamma_{k}\right) I_{k} \\
& =\sum_{j=1}^{n}\left(\sum_{k=1}^{n} a_{k} \beta_{k j} K\right) I_{j}-\sum_{j=1}^{n}\left(\alpha_{j}+d_{j}^{I}+\gamma_{j}\right) I_{j} \\
& =\sum_{j=1}^{n}\left[\sum_{k=1}^{n} K a_{k} \beta_{k j}-\left(\alpha_{j}+d_{j}^{I}+\gamma_{k}\right)\right] I_{j} .
\end{aligned}
$$


We choose appropriate numbers $a_{k}, 1 \leq k \leq n$, such that $\sum_{k=1}^{n} K a_{k} \beta_{k j}-\left(\alpha_{j}+d_{j}^{I}+\gamma_{k}\right)=0$; then, $\mathscr{L} V \leq M$, where $M$ is a positive constant. Therefore,

$$
\mathrm{d} V \leq M \mathrm{~d} t+\sum_{k=1}^{n}\left[\sigma_{1 k}\left(E_{k}-1\right) \mathrm{d} B_{k}(t)+\sigma_{2 k}\left(I_{k}-1\right) \mathrm{d} W_{k}(t)\right] .
$$

Integrate both sides of (21) from 0 to $\tau_{c} \wedge T$ and take expectation; then,

$$
\begin{aligned}
& \mathbb{E} V\left(S_{k}\left(\tau_{c} \wedge T\right), E_{k}\left(\tau_{c} \wedge T\right), I_{k}\left(\tau_{c} \wedge T\right)\right) \\
& \quad \leq V\left(S_{k}(0), E_{k}(0), I_{k}(0)\right)+\mathbb{E} \int_{0}^{\tau_{c} \wedge T} M \mathrm{~d} t \\
& \quad \leq V\left(S_{k}(0), E_{k}(0), I_{k}(0)\right)+M T .
\end{aligned}
$$

Set $\Omega_{c}=\left\{\tau_{c} \leq T\right\}$; we have $\mathbb{P}\left(\Omega_{c}\right) \geq \epsilon$. Notice that, for every $\omega \in \Omega_{c}$, there exists at least one of $S\left(\tau_{c}, \omega\right)$, $E\left(\tau_{c}, \omega\right), I\left(\tau_{c}, \omega\right)$ which equals either $c$ or $1 / c$. Therefore,

$$
\begin{aligned}
& V\left(S_{k}\left(\tau_{c} \wedge T\right), E_{k}\left(\tau_{c} \wedge T\right), I_{k}\left(\tau_{c} \wedge T\right)\right) \\
& \quad \geq \min _{1 \leq k \leq n}\left\{c-a_{k}-a_{k} \ln \frac{c}{a_{k}}, \frac{1}{c}-a_{k}-a_{k} \ln \frac{1}{a_{k} c}\right\} \\
& \wedge(c-1-\ln c) \wedge\left(\frac{1}{c}-1-\ln \frac{1}{c}\right) .
\end{aligned}
$$

Combining (22) with (23), we can obtain that

$$
\begin{aligned}
V\left(S_{k}(0), E_{k}(0), I_{k}(0)\right)+M T & \geq \mathbb{E}\left[1_{\Omega_{c}(\omega)} V\left(S_{k}\left(\tau_{c} \wedge T\right), E_{k}\left(\tau_{c} \wedge T\right), I_{k}\left(\tau_{c} \wedge T\right)\right)\right] \\
& \geq €\left\{\min _{1 \leq k \leq n}\left(c-a_{k}-a_{k} \ln \frac{c}{a_{k}}, \frac{1}{c}-a_{k}-a_{k} \ln \frac{1}{a_{k} c}\right)\right. \\
& \left.\wedge(c-1-\ln c) \wedge\left(\frac{1}{c}-1-\ln \frac{1}{c}\right)\right\},
\end{aligned}
$$

where $1_{\Omega_{m}(\omega)}$ is the indicator function of $\Omega_{m}$. Letting $m \longrightarrow \infty$ leads to the contradiction that $\infty>V\left(S_{k}(0), E_{k}(0), I_{k}(0)\right)+M T=\infty$. So, $\tau_{e}=\infty$ a.s. The proof is completed.

Corollary 1. For $S_{k}, k=1,2 \cdots n$, in model (4), there exists a set of $M_{k}$ such that $S_{k} \leq M_{k}$. Furthermore, the set $\Gamma=\left\{S_{k}: S_{k}>0, S_{k} \leq\left(\Lambda_{k} / d_{k}^{S}\right)\right\}$ is the invariant set, that is to say, if the initial value $S_{k}(0) \in \Gamma$, then $S_{k}(t) \in \Gamma$, for $t \geq 0$ almost surely.

Proof. For the first equation of model (4), we have $d S_{k} \leq\left(\Lambda_{k}-d_{k}^{S} S_{k}\right) d t$. By the method of variation of constants, we get that

$$
S_{k}(t) \leq \frac{\Lambda_{k}}{d_{k}^{S}}+\left(S_{k}(0)-\frac{\Lambda_{k}}{d_{k}^{S}}\right) e^{-d_{k}^{S} t}
$$

If $S_{k}(0) \leq\left(\Lambda_{k} / d_{k}^{S}\right)$, then $S_{k}(t) \leq\left(\Lambda_{k} / d_{k}^{S}\right)$. If $S_{k}(0)>$ $\left(\Lambda_{k} / d_{k}^{S}\right)$, then $S_{k}(t) \leq S_{k}(0)$. Let $M_{k}=\max \left\{\left(\Lambda_{k} / d_{k}^{S}\right), S_{k}(0)\right\}$. The proof is complete.
The assumption $S_{k}(0) \leq\left(\Lambda_{k} / d_{k}^{S}\right)$ will be used in the rest of the paper.

\section{The Behavior of the Model with $R_{0}<1$}

In the deterministic SEIR model, $P_{0}$ is the disease-free equilibrium, and it is globally stable which means that the disease will die out with any initial value when $R_{0}<1$. In this section, we will discuss the asymptotic behavior of the stochastic model with $R_{0}<1$.

Theorem 2. Let $\left(S_{1}(t), E_{1}(t), I_{1}(t), \ldots, S_{n}(t), E_{n}(t), I_{n}(t)\right)$ be the solution to model (4) with the initial valueinitial value $\left(S_{1}(0), E_{1}(0), I_{1}(0), \ldots, S_{n}(0), E_{n}(0), I_{n}(0) \in \mathbb{R}_{+}^{3 n}\right.$. If $B=\left(\beta_{k j}\right)_{n \times n}$ is irreducible and $R_{0}=\rho\left(M_{0}\right)<1$, then $P_{0}$ is the unique equilibrium of model (4), and it is globally asymptotically stable in probability.

Proof. According to the assumption, $B$ is irreducible and nonnegative; then, by Lemma $2, M_{0}$ has a single eigenvalue $\rho\left(M_{0}\right)$ and a positive eigenvector $\omega=\left(\omega_{1}, \omega_{2}, \ldots, \omega_{n}\right)$ corresponding to $\rho\left(M_{0}\right)$ such that 


$$
\left(\omega_{1}, \omega_{1}, \ldots, \omega_{n}\right) M_{0}=\left(\omega_{1}, \omega_{1}, \ldots, \omega_{n}\right) \rho\left(M_{0}\right) .
$$

Let $V_{1}=\sum_{k=1}^{n}(1 / 2) a_{k}\left(\left(\Lambda_{k} / d_{k}^{S}\right)-S_{k}\right)^{2}$ and $V_{2}=\sum_{k=1}^{n} c_{k}$ $\left(E_{k}+\left(\left(\epsilon_{k}+d_{k}^{E}\right) / \epsilon_{k}\right) I_{k}\right)$, where $c_{k}=\omega_{k} \epsilon_{k} /\left(d_{k}^{E}+\epsilon_{k}\right)\left(\alpha_{k}\right.$
$\left.+d_{k}^{I}+\gamma_{k}\right)$ and $a_{k}$ will be determined later. Using Itồ's formula, we can obtain that

$$
\begin{aligned}
\mathscr{L} V_{1}= & -\sum_{k=1}^{n} a_{k}\left(\frac{\Lambda_{k}}{d_{k}^{S}}-S_{k}\right)\left[\Lambda_{k}-\sum_{j=1}^{n} \beta_{k j} f_{k j}\left(S_{k}, I_{j}\right)-d_{k}^{S} S_{k}\right] \\
\leq & -\sum_{k=1}^{n} a_{k} d_{k}^{S}\left(\frac{\Lambda_{k}}{d_{k}^{S}}-S_{k}\right)^{2}+\sum_{k=1}^{n} \sum_{j=1}^{n} a_{k} \beta_{k j} C_{k j}\left(S_{k}\right) I_{j}\left(S_{k}-\frac{\Lambda_{k}}{d_{k}^{S}}\right) \\
\leq & -\sum_{k=1}^{n} a_{k} d_{k}^{S}\left(\frac{\Lambda_{k}}{d_{k}^{S}}-S_{k}\right)^{2}+\frac{\epsilon}{2} \sum_{k=1}^{n} \sum_{j=1}^{n} a_{k} \beta_{k j} C_{k j}\left(S_{k}^{0}\right)\left(\frac{\Lambda_{k}}{d_{k}^{S}}-S_{k}\right)^{2} \\
& +\frac{1}{2 \epsilon} \sum_{k=1}^{n} \sum_{j=1}^{n} a_{k} \beta_{k j} C_{k j}\left(S_{k}^{0}\right) I_{j}^{2} \\
= & -\sum_{k=1}^{n} a_{k}\left[d_{k}^{S}-\frac{\epsilon}{2} \sum_{j=1}^{n} \beta_{k j} C_{k j}\left(S_{k}^{0}\right)\right]\left(\frac{\Lambda_{k}}{\left.d_{k}^{S}-S_{k}\right)^{2}}\right. \\
& +\frac{1}{2 \epsilon} \sum_{k=1}^{n} \sum_{j=1}^{n} a_{k} \beta_{k j} C_{k j}\left(S_{k}^{0}\right) I_{j}^{2} .
\end{aligned}
$$

Here, the second inequality holds true because of the inequality $a b \leq(\epsilon / 2) a^{2}+(1 / 2 \epsilon) b^{2}$. Similarly, we use Itồ's formula to $V_{2}$ to get

$$
\begin{aligned}
\mathscr{L} V_{2} & =\sum_{k=1}^{n} c_{k}\left[\sum_{j=1}^{n} \beta_{k j} f_{k j}\left(S_{k}, I_{j}\right)-\frac{\left(\epsilon_{k}+d_{k}^{E}\right)\left(\alpha_{k}+d_{k}^{I}+\gamma_{k}\right)}{\epsilon_{k}} I_{k}\right] \\
& \leq \sum_{j=1}^{n} \sum_{k=1}^{n} \omega_{k} \frac{\epsilon_{k} \beta_{k j} C_{k j}\left(S_{k}^{0}\right)}{\left(\epsilon_{k}+d_{k}^{E}\right)\left(\alpha_{k}+d_{k}^{I}+\gamma_{k}\right)} I_{j}-\sum_{k=1}^{n} \omega_{k} I_{k} \\
& =\left(\rho_{0}-1\right) \sum_{k=1}^{n} \omega_{k} I_{k} .
\end{aligned}
$$

Define Lyapunov function $V(t)$ by $V(t)=V_{1}(t)+V_{2}(t)$, and according to Theorem $1, V(t)$ is positive definite; then,

$$
\begin{aligned}
\mathscr{L} V \leq & -\sum_{k=1}^{n} a_{k}\left[d_{k}^{S}-\frac{\epsilon}{2} \sum_{j=1}^{n} \beta_{k j} C_{k j}\left(S_{k}^{0}\right)\right]\left(\frac{\Lambda_{k}}{d_{k}^{S}}-S_{k}\right)^{2} \\
& +\frac{1}{2 \epsilon} \sum_{k=1}^{n} \sum_{j=1}^{n} a_{k} \beta_{k j} C_{k j}\left(S_{k}^{0}\right) I_{j}^{2}+\left(\rho_{0}-1\right) \sum_{k=1}^{n} \omega_{k} I_{k} .
\end{aligned}
$$

We can choose small $\epsilon$ such that $d_{k}^{S}-(\epsilon / 2) \sum_{j=1}^{n} \beta_{k j}$ $C_{k j}\left(S_{k}^{0}\right)>0, a_{k}$ are chosen to be sufficiently small, and because of $R_{0}<1$, we have $\mathscr{L} V<0$. Hence, applying Lemma 1 , we arrive at the desired assertion. The proof is completed.

\section{The Influence of Large Noise on Disease}

In this section, we will discuss the influence of large noises on disease when $R_{0}>1$. Before we give the theorem, an inequality is presented first.

Lemma 4. For $a_{k}, b_{k}, c_{k}, d_{k}, k=1,2 \cdots, n$, the following inequality holds true:

$$
\left[\sum_{k=1}^{n}\left(a_{k} b_{k}+c_{k} d_{k}\right)\right]^{2} \leq\left(\sum_{k=1}^{n} a_{k}^{2}+\sum_{k=1}^{n} c_{k}^{2}\right)\left(\sum_{k=1}^{n} b_{k}^{2}+\sum_{k=1}^{n} d_{k}^{2}\right) .
$$

Proof. We prove it by transforming it into an inner product in space $\mathbb{R}^{n}$. Let $\mathbf{a}:=\left(a_{1}, a_{2}, \ldots, a_{n}\right)^{T} \in \mathbb{R}^{n}$, and the vectors $\mathbf{b}, \mathbf{c}$, and $\mathbf{d}$ are defined in a similar way. Then,

$$
\begin{aligned}
\left(\mathbf{a}^{T} \mathbf{b}+\mathbf{c}^{T} \mathbf{d}\right)^{2}= & ((\mathbf{a}, \mathbf{b})+(\mathbf{c}, \mathbf{d}))^{2} \leq\|\mathbf{a}\|^{2}\|\mathbf{b}\|^{2}+\|\mathbf{c}\|^{2}\|\mathbf{d}\|^{2} \\
& +2\|\mathbf{a}\|\|\mathbf{b}\|\|\mathbf{c}\|\|\mathbf{d}\| \\
\leq & \|\mathbf{a}\|^{2}\|\mathbf{b}\|^{2}+\|\mathbf{c}\|^{2}\|\mathbf{d}\|^{2}+\|\mathbf{a}\|^{2}\|\mathbf{c}\|^{2}+\|\mathbf{b}\|^{2}\|\mathbf{d}\|^{2} \\
\leq & \left(\|\mathbf{a}\|^{2}+\|\mathbf{c}\|^{2}\right)\left(\|\mathbf{b}\|^{2}+\|\mathbf{d}\|^{2}\right) .
\end{aligned}
$$

The proof is completed.

Theorem 3. If $B=\left(\beta_{k j}\right)_{1 \leq k, j \leq n}$ is irreducible, then we have 


$$
\begin{aligned}
\max _{1 \leq k \leq n} & \left\{\lim \sup _{t \longrightarrow \infty} \frac{1}{t} \ln E_{k}(t), \lim \sup _{t \longrightarrow \infty} \frac{1}{t} \ln I_{k}(t)\right\} \\
\leq & \left(R_{0}-1\right) \max _{1 \leq k \leq n}\left\{\alpha_{k}+d_{k}^{I}+\gamma_{k}\right\} \\
& -\frac{1}{2 \sum_{i=1}^{n}\left(\left(1 / \sigma_{1 k}^{2}\right)+\left(1 / \sigma_{2 k}^{2}\right)\right)}, \quad \text { a.s. }
\end{aligned}
$$

Proof. We define a $C^{2}$ function $V\left(E_{k}, I_{k}\right)$ by

$$
V=\sum_{k=1}^{n} c_{k}\left(E_{k}+\frac{d_{k}^{E}+\epsilon_{k}}{\epsilon_{k}} I_{k}\right)
$$

where $c_{k}=\omega_{k} \epsilon_{k} /\left(d_{k}^{E}+\epsilon_{k}\right)\left(\alpha_{k}+d_{k}^{I}+\gamma_{k}\right)$. By calculation, we can get that

$$
\begin{aligned}
d V= & {\left[\sum_{k=1}^{n} \sum_{j=1}^{n} c_{k} \beta_{k j} f_{k j}\left(S_{k}, I_{j}\right)-\sum_{k=1}^{n} \omega_{k} I_{k}\right] \mathrm{d} t } \\
& +\sum_{k=1}^{n} c_{k} \sigma_{1 k} E_{k} \mathrm{~d} B_{k}(t) \\
& +\sum_{k=1}^{n} c_{k} \sigma_{2 k} \frac{d_{k}^{E}+\epsilon_{k}}{\epsilon_{k}} I_{k} \mathrm{~d} W_{k}(t) .
\end{aligned}
$$

Using Ito's formula, we arrive at

$$
\begin{aligned}
d \ln V= & \frac{1}{V}\left[\sum_{k=1}^{n} \sum_{j=1}^{n} c_{k} \beta_{k j} f_{k j}\left(S_{k}, I_{j}\right)-\sum_{k=1}^{n} \omega_{k} I_{k}\right] \mathrm{d} t \\
& -\frac{1}{2 V^{2}} \sum_{k=1}^{n} c_{k}^{2}\left[\sigma_{1 k}^{2} E_{k}^{2}+\sigma_{2 k}^{2} \frac{\left(d_{k}^{E}+\epsilon_{k}\right)^{2}}{\epsilon_{k}^{2}} I_{k}^{2}\right] \mathrm{d} t \\
& +\frac{1}{V} \sum_{k=1}^{n} c_{k} \sigma_{1 k} E_{k} d B_{k}(t)+\frac{1}{V} \sum_{k=1}^{n} c_{k} \sigma_{2 k} \frac{d_{k}^{E}+\epsilon_{k}}{\epsilon_{k}} I_{k} \mathrm{~d} W_{k}(t) . \\
\leq & \frac{1}{V}\left[\sum_{k=1}^{n} \sum_{j=1}^{n} c_{k} \beta_{k j} C_{k j}\left(S_{k}^{0}\right) I_{j}-\sum_{k=1}^{n} \omega_{k} I_{k}\right] d t \\
& -\frac{1}{2 V^{2}} \sum_{k=1}^{n} c_{k}^{2}\left[\sigma_{1 k}^{2} E_{k}^{2}+\sigma_{2 k}^{2} \frac{\left(d_{k}^{E}+\epsilon_{k}\right)^{2}}{\epsilon_{k}^{2}} I_{k}^{2}\right] \mathrm{d} t \\
& +\frac{1}{V} \sum_{k=1}^{n} c_{k} \sigma_{1 k} E_{k} \mathrm{~d} B_{k}(t)+\frac{1}{V} \sum_{k=1}^{n} c_{k} \sigma_{2 k} \frac{d_{k}^{E}+\epsilon_{k}}{\epsilon_{k}} I_{k} \mathrm{~d} W_{k}(t) . \\
=: & V_{1}(t) \mathrm{d} t+V_{2}(t) \mathrm{d} t+V_{3}(t)+V_{4}(t) .
\end{aligned}
$$

For $V_{1}(t)$, from the expression of eigenvector of $R_{0}$, i.e., $\left(\omega_{1}, \omega_{1} \cdots \omega_{n}\right) M_{0}=R_{0}\left(\omega_{1}, \omega_{1} \cdots \omega_{n}\right)$, we obtain that

$$
\begin{aligned}
V_{1}(t) & =\frac{1}{V}\left(R_{0}-1\right) \sum_{k=1}^{n} \omega_{k} I_{k} \\
& \leq \frac{R_{0}-1}{\sum_{k=1}^{n} c_{k}\left(d_{k}^{E}+\epsilon_{k} / \epsilon_{k}\right) I_{k}} \sum_{k=1}^{n} \omega_{k} I_{k} \\
& \leq \max _{1 \leq k \leq n}\left\{\alpha_{k}+d_{k}^{I}+\gamma_{k}\right\}\left(R_{0}-1\right) .
\end{aligned}
$$

According to the expression of $V$, utilizing Lemma 4 yields

$$
\begin{aligned}
V^{2} & =\left[\sum_{k=1}^{n}\left(c_{k} \sigma_{1 k} E_{k} \frac{1}{\sigma_{1 k}}+c_{k} \frac{\sigma_{2 k}\left(d_{k}^{E}+\epsilon_{k}\right) I_{k}}{\epsilon_{k}} \frac{1}{\sigma_{2 k}}\right)\right]^{2} \\
& \leq\left[\sum_{k=1}^{n} c_{k}^{2}\left(\sigma_{1 k}^{2} E_{k}^{2}+\frac{\sigma_{2 k}^{2}\left(d_{k}^{E}+\epsilon_{k}\right)^{2} I_{k}^{2}}{\epsilon_{k}^{2}}\right)\right]\left[\sum_{k=1}^{n}\left(\frac{1}{\sigma_{1 k}^{2}}+\frac{1}{\sigma_{2 k}^{2}}\right)\right] .
\end{aligned}
$$

Hence, $V_{2}(t)$ satisfies the inequality

$$
V_{2}(t) \leq \frac{1}{2 \sum_{k=1}^{n}\left(\left(1 / \sigma_{1 k}^{2}\right)+\left(1 / \sigma_{2 k}^{2}\right)\right)} .
$$

Because

$$
\limsup _{\mathrm{t} \longrightarrow \infty} \frac{1}{\mathrm{t}} \int_{0}^{\mathrm{t}} \frac{\sum_{\mathrm{k}=1}^{\mathrm{n}} \mathrm{c}_{\mathrm{k}}^{2} \sigma_{1 \mathrm{k}}^{2} \mathrm{E}_{\mathrm{k}}^{2}}{\mathrm{~V}^{2}} d t<\infty,
$$

applying Lemma 3 to $V_{3}(t)$ yields

$$
\limsup _{\mathrm{t} \longrightarrow \infty} \frac{1}{\mathrm{t}} \int_{0}^{\mathrm{t}} \sum_{\mathrm{k}=1}^{\mathrm{n}} \mathrm{V}^{-1} \mathrm{c}_{\mathrm{k}} \sigma_{1 \mathrm{k}} \mathrm{E}_{\mathrm{k}} \mathrm{dB}_{\mathrm{k}}(\mathrm{t})=0 .
$$

$V_{4}(t)$ can be done in the same way. Therefore,

$$
\begin{aligned}
\underset{\mathrm{l} \longrightarrow \infty}{\limsup } \frac{\ln \mathrm{V}(\mathrm{t})}{\mathrm{t}} \leq & \max _{1 \leq \mathrm{k} \leq \mathrm{n}}\left\{\mathrm{d}_{\mathrm{k}}^{\mathrm{I}}+\gamma_{\mathrm{k}}\right\}\left(\mathrm{R}_{0}-1\right) \\
& -\frac{1}{2 \sum_{\mathrm{k}=1}^{\mathrm{n}}\left(\left(1 / \sigma_{1 \mathrm{k}}^{2}\right)+\left(1 / \sigma_{2 \mathrm{k}}^{2}\right)\right)} .
\end{aligned}
$$

Since

$$
\begin{aligned}
\frac{1}{t} \ln \left(\sum_{k=1}^{n} c_{k} E_{k}\right) & \leq \frac{1}{t}\left[\ln \left(\max _{1 \leq k \leq n}\left\{c_{k}\right\} n \max _{1 \leq k \leq n}\left\{E_{k}\right\}\right)\right] \\
& \leq \frac{1}{t}\left[\ln \left(\max _{1 \leq k \leq n}\left\{c_{k}\right\} n\right)+\ln \left(\max _{1 \leq k \leq n}\left\{E_{k}\right\}\right)\right], \\
\frac{1}{t} \ln \left(\sum_{k=1}^{n} c_{k} E_{k}\right) & \geq \frac{1}{t}\left[\ln \left(\min _{1 \leq k \leq n}\left\{c_{k}\right\} \max _{1 \leq k \leq n}\left\{E_{k}\right\}\right)\right] \\
& \geq \frac{1}{t}\left[\ln \left(\min _{1 \leq k \leq n}\left\{c_{k}\right\}\right)+\ln \left(\max _{1 \leq k \leq n}\left\{E_{k}\right\}\right)\right],
\end{aligned}
$$

taking the upper limit yields 


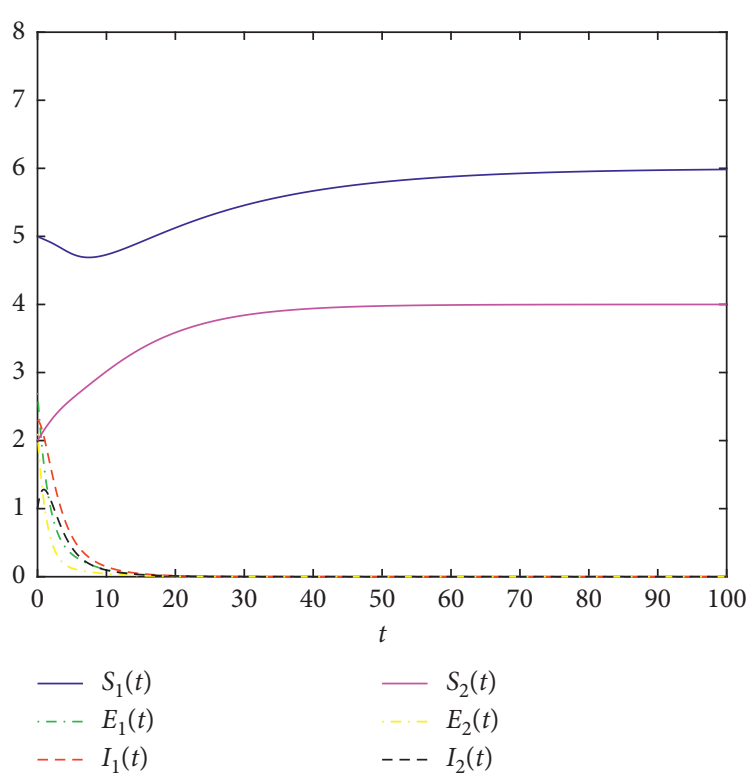

(a)
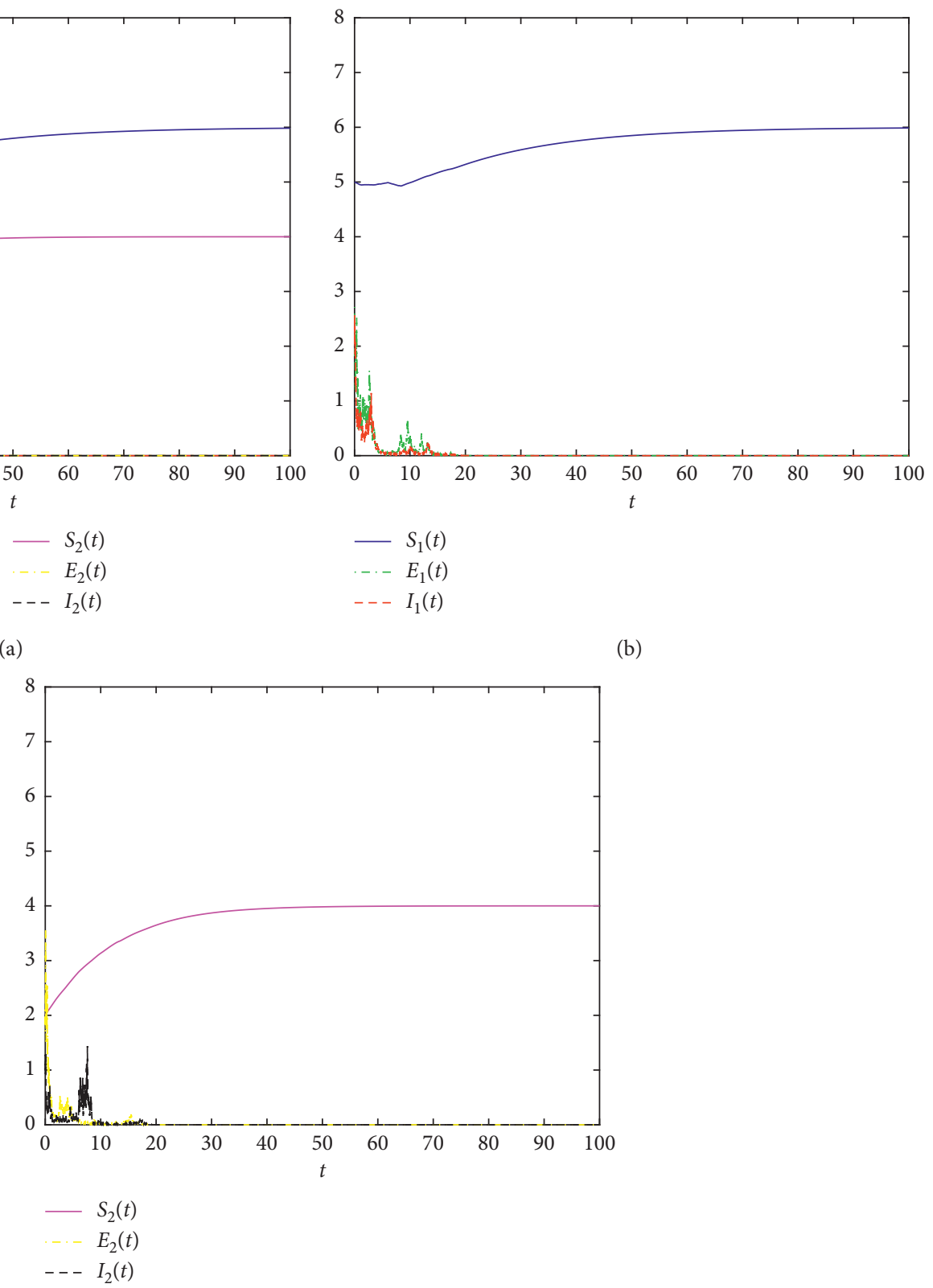

(b)

(c)

Figure 1: The trajectories with $R_{0}<1$ and initial value $S_{1}(0)=5, E_{1}(0)=2.7, I_{1}(0)=2.3 ; S_{2}(0)=2, E_{2}(0)=2.1, I_{2}(0)=1$ : (a) the trajectory without stochastic perturbation; $(b, c)$ the trajectories with stochastic perturbations where parameters are shown in Example 1.

$$
\limsup _{t \rightarrow \infty} \frac{1}{t} \ln \left(\sum_{k=1}^{n} c_{k} E_{k}\right)=\max _{1 \leq k \leq n}\left\{\limsup _{t \rightarrow \infty} \frac{1}{t} \ln E_{k}\right\} .
$$

Making use of the same method, we can obtain that $\underset{\mathrm{t} \longrightarrow \infty}{\limsup } \frac{1}{\mathrm{t}} \ln \left(\sum_{\mathrm{k}=1}^{\mathrm{n}} \frac{\mathrm{c}_{\mathrm{k}}\left(\mathrm{d}_{\mathrm{k}}^{\mathrm{E}}+\epsilon_{\mathrm{k}}\right)}{\epsilon_{\mathrm{k}}} \mathrm{I}_{\mathrm{k}}\right)=\max _{1 \leq \mathrm{k} \leq \mathrm{n}}\left\{\limsup _{\mathrm{t} \longrightarrow \infty} \frac{1}{t} \ln \mathrm{I}_{\mathrm{k}}\right\}$.

\section{Combining (43) and (44) yields}

$$
\underset{t \longrightarrow \infty}{\limsup } \frac{1}{t} \ln V(t) \geq \max _{1 \leq k \leq n}\left\{\limsup _{t \longrightarrow \infty} \frac{1}{t} \ln E_{k}, \underset{t \longrightarrow \infty}{\limsup } \frac{1}{t} \ln I_{k}\right\} .
$$

Along with (41), we arrive at the desired assertion. The proof is complete. 


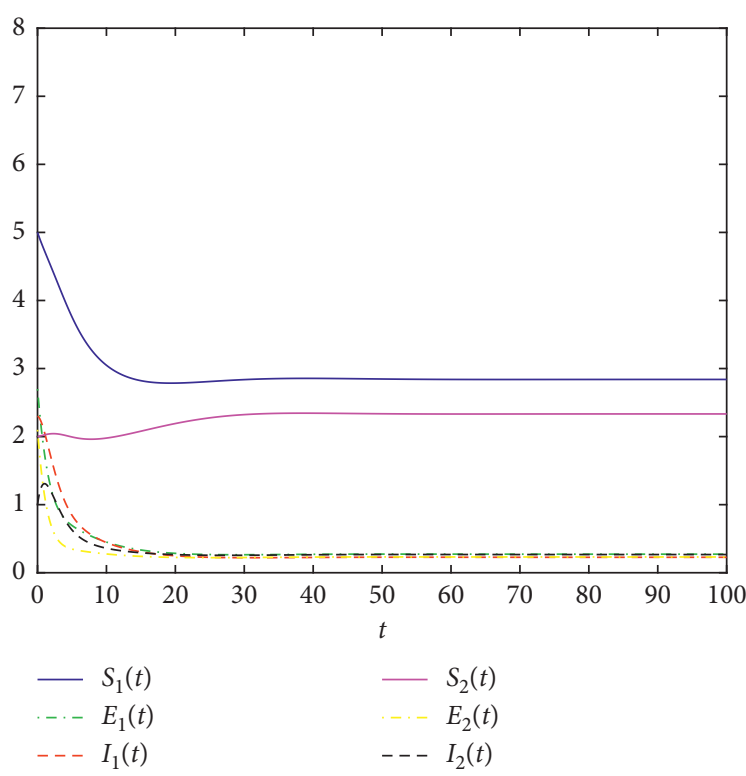

(a)
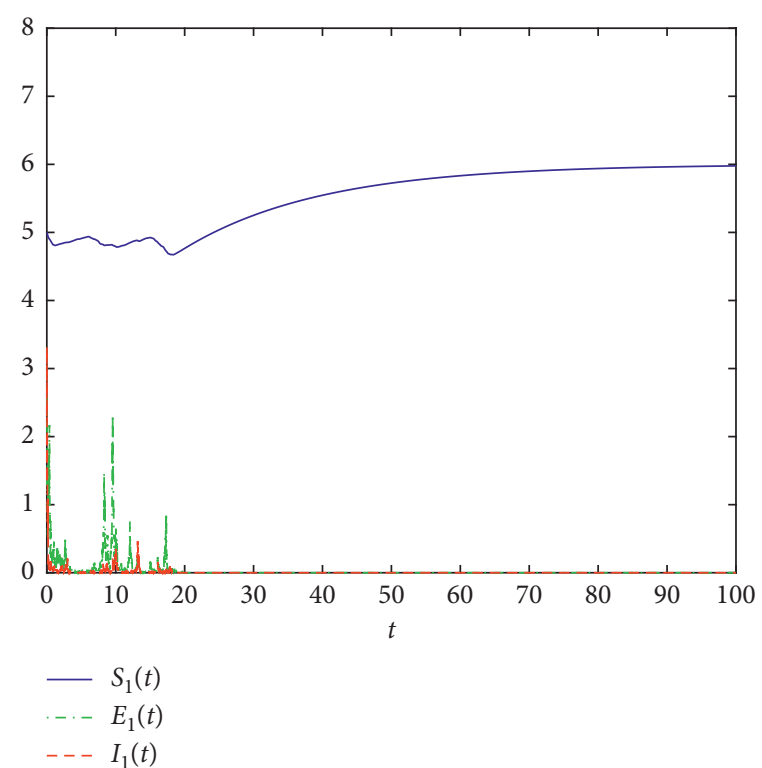

(b)

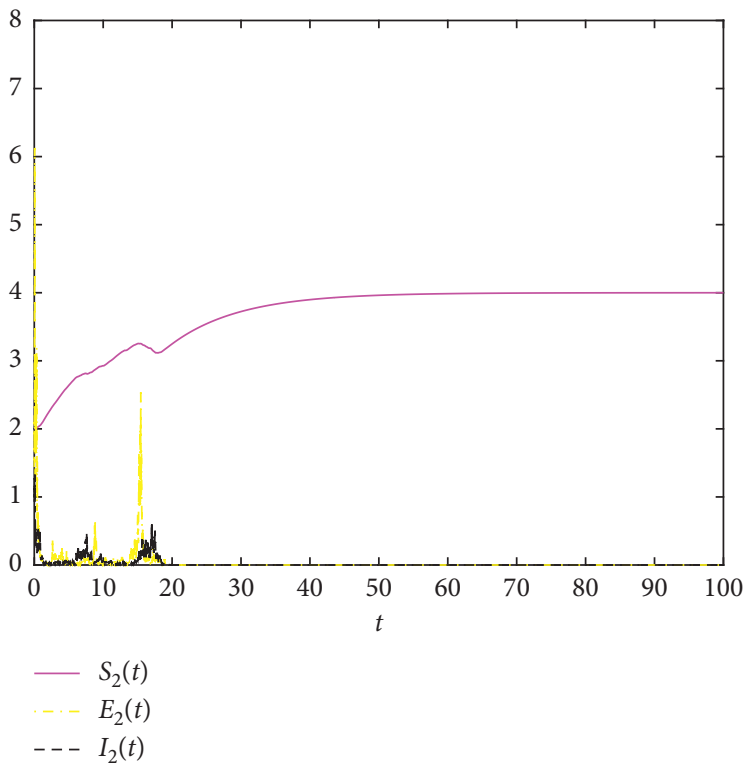

(c)

FIgURE 2: The trajectories with $R_{0}>1$ : (a) the trajectory without stochastic perturbation; (b, c) the trajectories with stochastic perturbations where parameters are shown in Example 2.

Corollary 2. For the solution to model (4), $E_{k}(t)$ and $I_{k}(t)$, $k=1,2 \cdots n$, decay exponentially to zero almost surely if

$$
\left(R_{0}-1\right) \max _{1 \leq \mathrm{k} \leq \mathrm{n}}\left\{\alpha_{k}+d_{k}^{I}+\gamma_{k}\right\}<\frac{1}{2 \sum_{i=1}^{n}\left(\left(1 / \sigma_{1 k}^{2}\right)+\left(1 / \sigma_{2 k}^{2}\right)\right)}
$$

Remark 2. From (46), we know that the right side of the inequality increases with the increase of $\sigma_{1 k}$ and $\sigma_{2 k}$; therefore, the inequality above holds true for certain $\alpha_{k}, d_{k}^{I}$, $\gamma_{k}$, and sufficiently large $\sigma_{1 k}$ and $\sigma_{2 k}$ even if $R_{0}>1$, which makes the disease extinct. It reflects that stochastic perturbations play an important role in disease control. Compared with the deterministic model in [4], the SEIR model with stochastic perturbations can show more properties and different behaviors.

Remark 3. We can see from many literature studies that the incidence function of the multigroup SEIR model is single one, such as $S_{k}(t) I_{j}(t)$ in $[5,7]$ and $S_{k}(t) I_{j}(t) /\left(1+\alpha_{k} I_{j}(t)\right)$ in [9]. These may have some limitations and cannot reflect the actual situation well. Incidence functions $f_{k j}\left(S_{k}(t), I_{j}(t)\right)$ in this paper can be expressed in different forms, which can better 

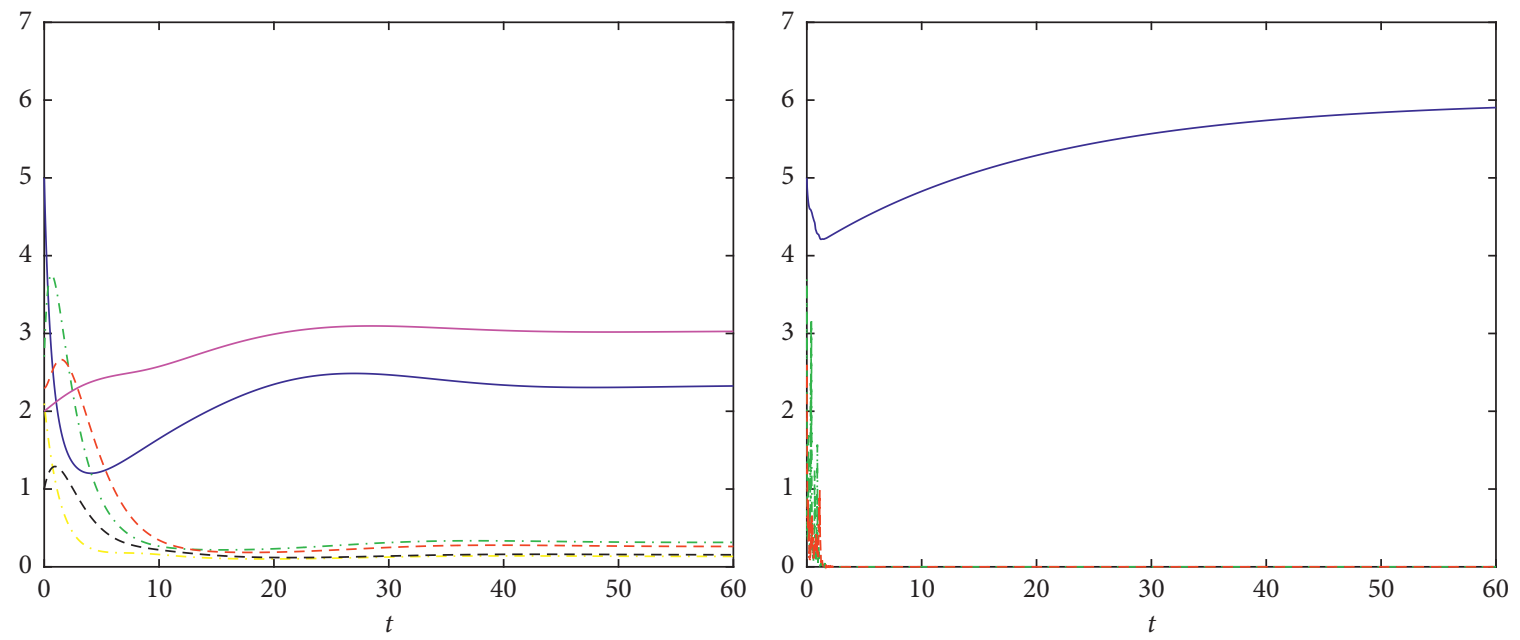

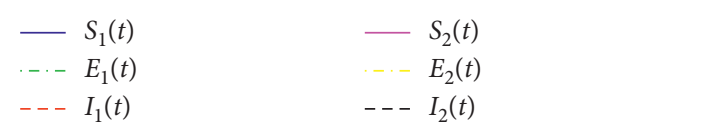

(a)

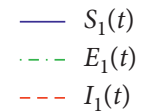

(b)

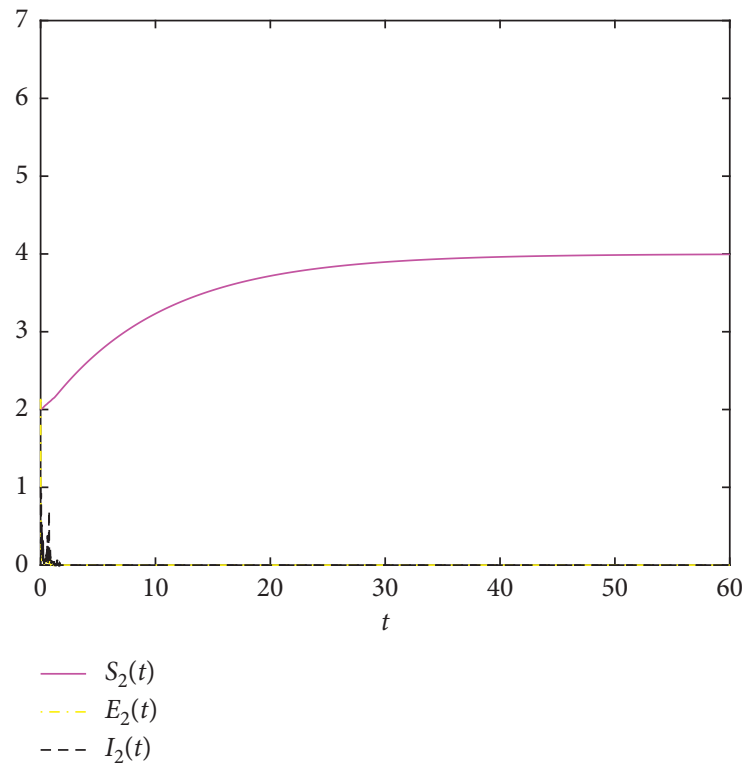

(c)

FIgURE 3: The trajectories with $R_{0}>1$ and two different incidence functions: (a) the trajectory without stochastic perturbation; (b, c) the trajectories with stochastic perturbations where parameters are shown in Example 3.

describe the reality of life. We will provide different examples to illustrate the results in Section 6 .

\section{Examples and Numerical Simulations}

In this section, we give some simulations of model (4) to confirm the analytical results above. By using Milstein's higher-order method [25], we obtain the corresponding discretization equation: 


$$
\begin{aligned}
& S_{i, k+1}=S_{i, k}+\left(\Lambda_{i}-\sum_{j=1}^{n} \beta_{i j} f_{k j}\left(S_{i, k}, I_{j, k}\right)-d_{i}^{S} S_{i, k}\right) \Delta t, \\
& E_{i, k+1}=E_{i, k}+\left(\sum_{j=1}^{n} \beta_{i j} f_{k j}\left(S_{i, k}, I_{j, k}\right)-\left(\epsilon_{i}+d_{i}^{E}\right) E_{i, k}\right) \Delta t+\sigma_{1 i} E_{i, k} \eta_{i, k} \sqrt{\Delta t} \\
& +\frac{1}{2} \sigma_{1 i}^{2} E_{i, k}\left(\eta_{i, k}^{2} \Delta t-\Delta t\right), \\
& I_{i, k+1}=I_{i, k}+\left(\epsilon_{i} E_{i, k}-\left(\alpha_{i}+d_{i}^{I}+\gamma_{i}\right) I_{i, k}\right) \Delta t+\sigma_{2 i} I_{i, k} \rho_{i, k} \sqrt{\Delta t}+\frac{1}{2} \sigma_{2 i}^{2} I_{i, k}\left(\rho_{i, k}^{2} \Delta t-\Delta t\right), \\
& \text { 0, 1). Let } n=2 \text {, i.e., we consider the } \\
& \text { ses in two groups. } \\
& \text { example to verify Theorem 2. } \\
& M_{0}=\left(\begin{array}{l}
\frac{\beta_{11} \epsilon_{1}\left(\Lambda_{1} / d_{1}^{S}\right)^{2}}{\left(\alpha_{1}+d_{1}^{I}+\gamma_{1}\right)\left(\epsilon_{1}+d_{1}^{E}\right)} \frac{\beta_{12} \epsilon_{1}\left(\Lambda_{1} / d_{1}^{S}\right)^{2}}{\left(\alpha_{1}+d_{1}^{I}+\gamma_{1}\right)\left(\epsilon_{1}+d_{1}^{E}\right)} \\
\frac{\beta_{21} \epsilon_{2}\left(\Lambda_{2} / d_{2}^{S}\right)}{\left(\alpha_{2}+d_{2}^{I}+\gamma_{2}\right)\left(\epsilon_{2}+d_{2}^{E}\right)} \frac{\beta_{22} \epsilon_{2}\left(\Lambda_{2} / d_{2}^{S}\right)}{\left(\alpha_{2}+d_{2}^{I}+\gamma_{2}\right)\left(\epsilon_{2}+d_{2}^{E}\right)}
\end{array}\right) .
\end{aligned}
$$

where $\eta_{i k}, \rho_{i k}$ are Gaussian random variables which follow the distribution $N(0,1)$. Let $n=2$, i.e., we consider the interactions of diseases in two groups.

First, we give an example to verify Theorem 2 .

Example 1. Assume that $f_{k j}=S_{k} I_{j} /\left(1+2 I_{j}^{2}\right)$. We choose $\Lambda_{1}=0.3, \Lambda_{2}=0.4 ; \epsilon_{1}=0.5, \epsilon_{2}=0.6 ; \gamma_{1}=0.4, \gamma_{2}=0.3 ; \alpha_{1}=$ $0.1, \alpha_{2}=0.07 ; \beta_{11}=0.02, \beta_{12}=0.05, \beta_{21}=0.04, \beta_{22}=0.02$, $d_{1}^{S}=0.05, d_{2}^{S}=0.1, d_{1}^{E}=0.08, d_{2}^{E}=0.12, d_{1}^{I}=0.1, d_{2}^{I}=0.15$ such that $R_{0}=0.4835<1$, which satisfies the condition of Theorem 2. Moreover, let $\sigma_{11}=1, \sigma_{12}=0.5, \sigma_{21}=0.8$, and $\sigma_{22}=1.5$. Its trajectory is shown in Figure 1 .

From Figure 1(a), we can see that the diseases are extinct when stochastic perturbations are absent. From Figures 1(b) and $1(\mathrm{c})$, we can see the diseases in two groups are globally asymptotically stable.

Now, we move forward to verify Theorem 3 . We will present two examples to illustrate the two cases of incidence functions. In Example 2, we give the same incidence function for two groups, and in Example 3, two different incidence functions are presented.

Example 2. Assume that $f_{k j}=S_{k} I_{j} /\left(1+2 I_{j}^{2}\right)$. We choose $\Lambda_{1}=0.3, \Lambda_{2}=0.4 ; \epsilon_{1}=0.5, \epsilon_{2}=0.6 ; \gamma_{1}=0.4, \gamma_{2}=0.3 ; \alpha_{1}=0.1$, $\alpha_{2}=0.07 ; \beta_{11}=0.1, \beta_{12}=0.05, \beta_{21}=0.12, \beta_{22}=0.2, d_{1}^{S}=0.05$, $d_{2}^{S}=0.1, d_{1}^{E}=0.08, d_{2}^{E}=0.12, d_{1}^{I}=0.1, d_{2}^{I}=0.15$ such that $R_{0}=1.685>1$, which satisfies the condition of Theorem 3 . Moreover, let $\sigma_{11}=1, \sigma_{12}=0.5, \sigma_{21}=0.8, \sigma_{22}=1.5$ so that $\left(R_{0}-1\right) \max _{1 \leq k \leq n}\left\{\alpha_{k}+d_{k}^{I}+\gamma_{k}\right\}<1 / 2 \sum_{i=1}^{n}\left(\left(1 / \sigma_{1 k}^{2}\right)+\left(1 / \sigma_{2 k}^{2}\right)\right)$ is satisfied. Its trajectory is shown in Figure 2. From Figure 2(a), we can see that the diseases are persistent because of $R_{0}>1$ when stochastic perturbation is absent. We can see in Figures 2(b) and 2(c) that the diseases in two groups die out under certain stochastic perturbations and the exposed are the same results.

Example 3. Assume that $f_{1 j}=S_{1}^{2} I_{j}, f_{2 j}=S_{k} I_{j} /\left(1+2 I_{j}^{2}\right)$, $j=1,2$, such that
We choose $\quad \Lambda_{1}=0.3, \Lambda_{2}=0.4 ; \epsilon_{1}=0.5, \epsilon_{2}=0.6$; $\gamma_{1}=0.4, \gamma_{2}=0.3 ; \alpha_{1}=0.1, \alpha_{2}=0.07 ; \beta_{11}=0.04, \beta_{12}=0.15$, $\beta_{21}=0.1, \beta_{22}=0.05, d_{1}^{S}=0.05, d_{2}^{S}=0.1, d_{1}^{E}=0.08, d_{2}^{E}=0.12$, $d_{1}^{I}=0.1, d_{2}^{I}=0.15$ so that $R_{0}=3.61>1$ can be obtained. Moreover, let $\sigma_{11}=4, \sigma_{12}=4.5, \sigma_{21}=2.8$, and $\sigma_{22}=5$; then, the conditions in Theorem 3 are satisfied. Its trajectory is shown in Figure 3. From Figure 3(a), we can see that the diseases are persistent because $R_{0}>1$ without stochastic perturbation. We can see in Figures 3(b) and 3(c) that the exposed and infected in two groups die out under certain stochastic perturbations, which conform to the results of Theorem 3.

\section{Data Availability}

The data used to support the findings of this study are included within the article.

\section{Conflicts of Interest}

The authors declare that they have no conflicts of interest.

\section{Acknowledgments}

This work was supported by the National Natural Science Foundation of China (no. 11661065), the Fundamental Research Funds for the Central South University under grant no. 2018zzts099, and the Scientific Research Funds of Jiangxi Provincial Education Department (GJJ181105 and GJJ191145). 


\section{References}

[1] A. Lajmanovich and J. A. Yorke, "A deterministic model for gonorrhea in a nonhomogeneous population," Mathematical Biosciences, vol. 28, no. 3-4, pp. 221-236, 1976.

[2] C. Ji, D. Jiang, and N. Shi, "Multigroup SIR epidemic model with stochastic perturbation," Physica A: Statistical Mechanics and Its Applications, vol. 390, no. 10, pp. 1747-1762, 2011.

[3] M. Liu, C. Bai, and K. Wang, "Asymptotic stability of a twogroup stochastic SEIR model with infinite delays," Communications in Nonlinear Science and Numerical Simulation, vol. 19, no. 10, pp. 3444-3453, 2014.

[4] R. Sun and J. Shi, "Global stability of multigroup epidemic model with group mixing and nonlinear incidence rates," Applied Mathematics and Computation, vol. 218, no. 2, pp. 280-286, 2011.

[5] Q. Yang and X. Mao, "Extinction and recurrence of multigroup SEIR epidemic models with stochastic perturbations," Nonlinear Analysis: Real World Applications, vol. 14, no. 3, pp. 1434-1456, 2013.

[6] J. Yu, D. Jiang, and N. Shi, "Global stability of two-group SIR model with random perturbation," Journal of Mathematical Analysis and Applications, vol. 360, no. 1, pp. 235-244, 2009.

[7] C. Yuan, D. Jiang, D. O'Regan, and R. P. Agarwal, "Stochastically asymptotically stability of the multi-group SEIR and SIR models with random perturbation," Communications in Nonlinear Science and Numerical Simulation, vol. 17, no. 6, pp. 2501-2516, 2012.

[8] Z. Yuan and L. Wang, "Global stability of epidemiological models with group mixing and nonlinear incidence rates," Nonlinear Analysis: Real World Applications, vol. 11, no. 2, pp. 995-1004, 2010.

[9] Q.-X. Han and Z.-G. Wang, "On extinction of infectious diseases for multi-group SIRS models with saturated incidence rate," Advances in Difference Equations, vol. 2015, no. $333,2015$.

[10] Z. Liu, "Dynamics of positive solutions to SIR and SEIR epidemic models with saturated incidence rates," Nonlinear Analysis: Real World Applications, vol. 14, no. 3, pp. 12861299, 2013.

[11] Q. Yang, D. Jiang, N. Shi, and C. Ji, "The ergodicity and extinction of stochastically perturbed SIR and SEIR epidemic models with saturated incidence," Journal of Mathematical Analysis and Applications, vol. 388, no. 1, pp. 248-271, 2012.

[12] Q. Liu and D. Jiang, "Asymptotic behavior of a stochastic delayed SEIR epidemic model with nonlinear incidence," Physica A: Statistical Mechanics and Its Applications, vol. 462, pp. 870-882, 2016.

[13] Q. Shi, D.-Q. Jiang, and N.-Z. Shi, "Stationarity and periodicity of positive solutions to stochastic SEIR epidemic models with distributed delay," Discrete and Continuous Dynamical Systems-Series B, vol. 22, pp. 2479-2500, 2017.

[14] P. Driessche and J. Watmough, "Reproduction numbers and sub-threshold endemic equilibria for compartmental models of disease transmission," Mathematical Biosciences, vol. 180, pp. 29-48, 2002.

[15] J. Artalejo, A. Economou, and M. Lopez-Herrero, “The stochastic SEIR model before extinction: computational approaches," Applied Mathematics and Computation, vol. 265, pp. 2016-2043, 2015.

[16] A. Myr, A. Assadouq, L. Omari, A. Settati, and A. Lahrouz, "A stochastic SIR epidemic system with a nonlinear relapse," Discrete Dynamics in Nature and Society, vol. 2018, Article ID 5493270, 10 pages, 2018.
[17] F. Wang and Z. M. Liu, "Dynamical behavior of stochastic SIRS model with two different incidence rates and Markovian switching," Advances in Difference Equations, vol. 2019, no. $322,2019$.

[18] J. H. Bao and J. H. Shao, "Asymptotic behavior of SIRS models in state-dependent random environments," 2018, https:// arxiv.org/abs/1802.02309.

[19] X. Guo and J. Luo, "Stationary distribution and extinction of SIR model with nonlinear incident rate under Markovian switching," Physica A: Statistical Mechanics and Its Applications, vol. 505, pp. 471-481, 2018.

[20] D. Li, S. Liu, and J. a. Cui, "Threshold dynamics and ergodicity of an SIRS epidemic model with Markovian switching," Journal of Differential Equations, vol. 263, no. 12, pp. 88738915, 2017.

[21] N. Dalal, D. Greenhalgh, and X. Mao, "A stochastic model of AIDS and condom use," Journal of Mathematical Analysis and Applications, vol. 325, no. 1, pp. 36-53, 2007.

[22] P. J. Witbooi, "Stability of an SEIR epidemic model with independent stochastic perturbations," Physica A: Statistical Mechanics and Its Applications, vol. 392, no. 20, pp. 49284936, 2013.

[23] R.-Z. Hasminskii, Stochastic Stability of Differential Equations, Springer, Alphen aan den Rijn, The Netherlands, 1980.

[24] X.-R. Mao, Stochastic Differential Equation and Applications, Horwood, Chichester, UK, 1997.

[25] D. J. Higham, "An algorithmic introduction to numerical simulation of stochastic differential equations," Siam Review, vol. 43 , no. 3 , pp. 525-546, 2001. 